\title{
The Construction of the sh-Lie-Algebra of Closed Bosonic Strings
}

\author{
Hans-Werner Wiesbrock \\ Institut für Theorie der Elementarteilchen, Arnimalle 14, W-1000 Berlin 33, FRG
}

Received December 18, 1990

\begin{abstract}
We present a construction of the closed string algebra in terms of Gaussian processes and crossed products. Also we give a purely functional analytical prove of the sh-Lie-structure.
\end{abstract}

\section{Introduction}

In 1989 M. Saadi and B. Zwiebach, [S-Z], inspired by the work of M. Kaku, [K], introduced an interaction of closed string fields along polyhedra. This was further investigated and generalized by T. Kugo, H. Kunimoto and K. Suehiro, [K-K-S, $\mathrm{K}-\mathrm{S}]$. They called it a nonpolynomial closed string field theory. They presented the theory in the operator formalism using the techniques of conformal theory and complex analysis. The nonpolynomial interaction of closed string fields should obey a gauge invariance, which was reformulated as an algebraic property. This later property was proved in $[\mathrm{K}-\mathrm{S}]$ and for more general background fields in $[\mathrm{S}]$. It was J. Stasheff who recognized that these algebraic relations define a strongly homotopy Lie algebra (sh-Lie-algebra), [St1]. The aim of this paper is to construct this algebra of closed string fields by using techniques of functional analysis. Also we will give an analytic proof of the sh-Lie-structure.

\section{The Closed String Bosonic Fields}

A classical closed string in the Euclidean formulation is given by a continuous imbedding

$$
\omega: S^{1} \rightarrow \mathbf{R}^{d} .
$$

In the Schrödinger picture of quantization the closed string fields are functionals on such paths. The measure of integration is given by the free dynamics of the classical string. 
Let

$$
d \mu=\bigoplus_{i=1}^{d} d \mu_{L_{0}} \oplus d y,
$$

where $d \mu_{L_{0}}$ is the Gaussian measure with covariance $L_{0}^{-1}$,

$$
L_{0}=\sqrt{-\Delta_{p}^{\prime}}
$$

and $-\Delta_{p}$ is the Laplacian on $L^{2}([-\pi, \pi])$ with periodic boundary conditions in $-\pi, \pi,-\Delta_{p}^{\prime}$ the restriction of $-\Delta_{p}$ onto the orthogonal complement of the kernel (without zero modes). The kernels sum up to $\mathbf{R}^{d}$. Let $d y$ denote the Lebesgue measure on $\mathbf{R}^{d}$. We can split an arbitrary closed path into $\omega=\omega^{\prime}+y, y=$ orthogonal projection of $\omega$ onto the kernel, i.e., a based part and the localization of $\omega(0)$.

A closed string bosonic field $\Phi$ is then by definition a square integrable function w.r.t. $d \mu$.

In order to split strings etc. we have to take care.

Lemma 1. For $\mu_{L_{0}}$-almost every $\omega \in \mathscr{S}^{\prime}[-\pi, \pi]$ there exists no nonempty open set $U \subset[-\pi, \pi]$ such that $\omega$ restricted on $U$ is a signed measure. In particular, $\omega$ is not a continuous function on any such $U$.

Proof. The covariance of $\mu_{L_{0}}$

$$
\begin{aligned}
c(s, t) & =\sum_{n \in \mathbf{Z} \backslash 0} \frac{1}{|n|} e^{i n \pi t} e^{-i n \pi s} \\
& =\ln \left(1-e^{i \pi(t+s)}\right)\left(1-e^{i \pi(t-s)}\right)
\end{aligned}
$$

is obviously not continuous, which implies the lemma, [Co-La].

For the definition of the interaction we need the splitting of closed strings. By the lemma we cannot define this in a naive way. We need some technical preliminaries.

Let $\sigma_{0}=-\pi<\sigma_{1}<\cdots<\sigma_{n}=\pi, n \in \mathbf{N}$, be a partition of $[-\pi, \pi]$. Define

$$
L_{\sigma}:=L_{\sigma_{1}}+\cdots+L_{\sigma_{n}}+I_{1}+\cdots+I_{n} \text {, }
$$

where $L_{\sigma, i}=\sqrt{-\Delta_{N, i}^{\prime}}$ and $-\Delta_{N, i}$ the Neumann Laplacian on $L^{2}\left(\left[\sigma_{i-1}, \sigma_{i}\right]\right),-\Delta_{N, i}^{\prime}$ the restriction of $-\Delta_{N, i}$ on the complement of the kernel, see (33), $I_{i}=$ identity on $\operatorname{span}\left\{\chi_{\left[\sigma_{i-1}, \sigma_{i}\right]}\right\}$ ( $\chi$ the characteristic function). We want to prove

Theorem. 2. Let $n \in \mathbf{N}, \sigma_{i}, L_{\sigma}$ as above. Then

$$
L_{0}^{-1}-L_{\sigma}^{-1} \text { is of trace class. }
$$

This technical result will allow us to define appropriate splittings of closed strings needed for the interaction. For the proof of the theorem we use

Lemma 3. Let $[a, b] \subset \mathbf{R}$ be an interval, $-\Delta_{D}$ (respectively $\left.-\Delta_{N}^{\prime}\right)$ the Laplacian on $L^{2}([a, b])$ with Dirichlet (Neumann) boundary conditions at a and $b$ (without zero mode). Then

$$
\left(\sqrt{-\Delta_{N}^{\prime}}\right)^{-1}-\left(\sqrt{-\Delta_{D}}\right)^{-1}
$$

restricted onto the complement of the kernel of $-\Delta_{N}$ is of trace class. 
Assuming Lemma 3 we now give a

Proof of Theorem 2. Let $L_{\sigma_{1}}$ as above,

$$
-\Delta_{N, \sigma}=-\Delta_{N, 1}+\cdots+\left(-\Delta_{N, n}\right)
$$

and

$$
-\Delta_{D, \sigma}:=-\Delta_{D, 1}+\cdots+\left(-\Delta_{D, n}\right),
$$

the analog with Neumann boundary conditions replaced by Dirichlet boundary conditions.

In general we have $[\mathrm{R}-\mathrm{S}$ IV, p. 270]

$$
0 \leqq-\Delta_{N, \sigma} \leqq-\Delta_{N}
$$

where $-\Delta_{N}$ is the Neumann Laplacian on $L^{2}([-\pi, \pi])$, and

$$
0 \leqq-\Delta_{D} \leqq-\Delta_{D, \sigma}
$$

with $-\Delta_{D}$ the Dirichlet Laplacian on $L^{2}([-\pi, \pi])$. Furthermore we have in general [G-R-S, p. 254]

$$
0<-\Delta_{N}+\alpha \operatorname{Id} \leqq-\Delta_{p}+\alpha \operatorname{Id} \leqq-\Delta_{D}+\alpha \mathrm{Id}, \quad \alpha \in \mathbf{R}^{+} \backslash 0 .
$$

Denote $\mathscr{H}_{0}$ the closed subspace spanned by the zero modes of $-\Delta_{N, i}$, i.e.

$$
\mathscr{H}_{0}=\operatorname{span}\left\{\chi_{\left[\sigma_{i-1}, \sigma_{l}\right]}\right\},
$$

$P_{0}$ the orthogonal projection onto $\mathscr{H}_{0}^{\perp}$. Then we get

$$
\begin{aligned}
0 \leqq P_{0}\left(-\Delta_{N, \sigma}\right) P_{0} & \leqq P_{0}\left(-\Delta_{N}\right) P_{0} \leqq P_{0}\left(-\Delta_{p}\right) P_{0} \\
& \leqq P_{0}\left(-\Delta_{D}\right) P_{0} \leqq P_{0}\left(-\Delta_{D, \sigma}\right) P_{0} \leqq-\Delta_{D, \sigma}
\end{aligned}
$$

Taking inverse and square roots we get

$$
0 \leqq P_{0}\left(-\Delta_{D, \sigma}\right)^{-1 / 2} P_{0} \leqq P_{0}\left(-\Delta_{p}\right)^{-1 / 2} P_{0} \leqq P_{0}\left(-\Delta_{N, \sigma}\right)^{-1 / 2} P_{0}
$$

This shows the estimate

$$
0 \leqq P_{0}\left(\left(-\Delta_{N, \sigma}\right)^{-1 / 2}-\left(-\Delta_{p}\right)^{-1 / 2}\right) P_{0} \leqq P_{0}\left(-\Delta_{N, \sigma}\right)^{-1 / 2}-\left(-\Delta_{D, \sigma}\right)^{-1 / 2} P_{0} .
$$

Now

$$
\left(-\Delta_{N, \sigma}\right)^{-1 / 2}-\left(-\Delta_{D, \sigma}\right)^{-1 / 2}=\sum_{i=1}^{n}\left(-\Delta_{N, i}\right)^{-1 / 2}-\left(-\Delta_{D, i}\right)^{-1 / 2} .
$$

Using Lemma 3 we easily get the theorem.

So we are left with the

Proof of Lemma 3. Scaling the interval we can assume $[a, b]=[-1,1]$. Then

$$
\begin{aligned}
\phi_{2 n}(t) & :=\sqrt{2} \sin (\pi n t), \\
\phi_{2 n+1}(t) & :=\sqrt{2} \cos ((n+1 / 2) \pi t),
\end{aligned}
$$


$n \in \mathbf{N}$ is an orthonormal basis of eigenfunctions for $\left(-\Delta_{D}\right)$,

$$
\begin{aligned}
\psi_{2 n}(t) & :=\sqrt{2} \cos (\pi n t), \\
\psi_{2 n+1}(t) & :=\sqrt{2} \sin ((n+1 / 2) \pi t),
\end{aligned}
$$

an orthonormal basis of eigenvectors for $\left(-\Delta_{N}\right)$.

For their scalar products one computes

$$
\begin{array}{ll}
\left|\left\langle\psi_{n}, \phi_{m}\right\rangle\right|=0 & \text { for } m, n \text { odd or } m, n \text { even, } \\
\left|\left\langle\psi_{n}, \phi_{m}\right\rangle\right|=\frac{4 m}{\pi\left|n^{2}-m^{2}\right|} \quad \text { for } m \text { odd, } n=4 k, \text { or } n \text { odd, } m=4 k+2, k \in \mathbf{N}, \\
\left|\left\langle\psi_{n}, \phi_{m}\right\rangle\right|=\frac{4 n}{\pi\left|n^{2}-m^{2}\right|} \quad \text { for } n \text { odd, } m=4 k, \text { or } m \text { odd, } n=4 k+2 .
\end{array}
$$

By the general estimate on the Laplacians it is enough to show

$$
\sum_{n \geqq 1}\left(\frac{1}{n}-S_{n}\right)<\infty
$$

with

$$
S_{n}:=\left\langle\psi_{n},\left(-\Delta_{D}\right)^{1 / 2} \psi_{n}\right\rangle=\sum_{k=1}^{\infty}\left(\left\langle\phi_{n}, \psi_{k}\right\rangle\right)^{2} \cdot \frac{1}{k} .
$$

All summands are positive and we can rearrange the sum arbitrarily. Let us first prove a simple general estimate

Lemma 4. For $n \in \mathbf{N}$ we have

i)

$$
\sum_{k=0}^{\infty} \frac{2 k+1}{\left((2 n)^{2}-(2 k+1)^{2}\right)^{2}}=\frac{1}{2 n} \cdot \frac{\pi^{2}}{8}+O\left(\frac{1}{n^{2}}\right) .
$$

ii)

$$
\sum_{k=0}^{\infty} \frac{1}{\left((2 n)^{2}-(2 k+1)^{2}\right)(2 k+1)}=O\left(\frac{1}{n^{2}}\right) \text {. }
$$

Proof.

i)

$$
\begin{aligned}
\sum_{k=0}^{\infty} \frac{2 k+1}{\left((2 n)^{2}-(2 k+1)^{2}\right)^{2}} & =\frac{1}{8 n} \sum_{k=0}^{\infty}\left(\frac{-1}{(2 n+2 k+1)^{2}}+\frac{1}{(2 n-2 k-1)^{2}}\right) \\
& =\frac{1}{4 n}\left(\sum_{k=0}^{\infty} \frac{1}{(2 k+1)^{2}}-\sum_{k=n}^{\infty} \frac{1}{(2 k+1)^{2}}\right) \\
& =\frac{\pi^{2}}{8} \cdot \frac{1}{4 n}+O\left(\frac{1}{n^{2}}\right) .
\end{aligned}
$$

ii) $\sum_{k=0}^{\infty} \frac{1}{\left((2 n)^{2}-(2 k+1)^{2}\right)(2 k+1)}=\sum_{k=0}^{\infty} \frac{1}{(2 k+1)(2 n+2 k+1)(2 n-2 k-1)}$

$$
=\sum_{k=0}^{2 n}+\sum_{k=2 n+1} \frac{1}{(2 k+1)(2 n+2 k+1)(2 n-2 k-1)} \text {. }
$$


Using the integral criterion we estimate

$$
\sum_{k=2 n+1}^{\infty} \frac{1}{(2 k+1)(2 n+2 k+1)(2 n-2 k-1)} \leqq O\left(\frac{1}{n^{2}}\right) .
$$

Rearranging the first sum we get

$$
\begin{aligned}
& =\sum_{k=0}^{n} \frac{1}{(2 k+1)(2 n+2 k+1)}\left(\frac{1}{(2(n-k)-1)}-\frac{1}{(2(2 n+k)+1)}\right) \\
& =\sum_{k=0}^{n} \frac{2+2 n+3 k}{(2 k+1)(2(n+k)+1)(2(n-k)-1)(2(2 n+k)+1)} .
\end{aligned}
$$

Estimating

$$
\frac{2+2 n+3 k}{2(n+k)+1} \leqq \frac{3}{2}
$$

and applying the integral criterion shows the lemma.

Now we can estimate

$$
\begin{aligned}
S_{4 n} & =\sum_{k=0}^{\infty} \frac{1}{\pi^{2}}\left(\frac{4(2 k+1)}{(4 n)^{2}-(2 k+1)^{2}}\right)^{2} \cdot \frac{1}{2 k+1} \\
& =\frac{1}{4 n}
\end{aligned}
$$

and using

$$
\begin{aligned}
& \left(\frac{4 n+2}{(4 n+2)^{2}-(2 k+1)^{2}}\right)^{2} \frac{1}{2 k+1} \\
& \quad=\frac{16(2 k+1)}{\left((4 n+2)^{2}-(2 k+1)^{2}\right)^{2}}+\frac{16}{\left((4 n+2)^{2}-(2 k+1)^{2}\right)(2 k+1)}
\end{aligned}
$$

we get

$$
S_{4 n+2}=\frac{1}{4 n+2}+O\left(\frac{1}{n^{2}}\right)
$$

Similarly we get

$$
S_{2 n+1}=\frac{1}{2 n+1}+O\left(\frac{1}{n^{2}}\right)
$$

and thereby with

$$
\sum_{n=1}^{\infty}\left(\frac{1}{n}-S_{n}\right) \leqq O\left(\sum_{n=1}^{\infty} \frac{1}{n^{2}}\right)<\infty
$$

the final proof or the theorem.

Our main application of the theorem is

Corollary 5. Let $\sigma=\left\{\sigma_{i}\right\}_{i=0, ., n}, n \in \mathbf{N}$ be a partition of $[-\pi, \pi], d x_{i}$ the Lebesgue 
measure on the 1-dim. $\operatorname{span}\left\{\chi_{\left[\sigma_{i-1}, \sigma_{i}\right]}\right\}, i=1, ., n$. Then

$$
\mu_{L_{0}} \otimes d y\left(\omega^{\prime}, y\right)=F_{\sigma}\left(\omega_{1}^{\prime}, \ldots, \omega_{n}^{\prime}, x_{1}^{\prime}, \ldots, x_{n}^{\prime}\right) \bigotimes_{i=1}^{n} \mu_{L_{\sigma, i}}\left(\omega_{i}^{\prime}\right) \bigotimes_{i=1}^{n} d x_{i},
$$

with a nonvanishing measurable function $F_{\sigma}$, i.e. the two measures are absolutely continuous.

Proof. Noting that the two measures

$$
\mu_{L_{\sigma}}\left(\omega_{1}^{\prime}, ., \omega_{n}, x_{1}^{\prime}, ., x_{n}^{\prime}\right) \approx \bigotimes_{i=1}^{n} \mu_{L_{\sigma, i}}\left(\omega_{i}^{\prime}\right) \bigotimes_{i=1}^{n} d x_{i}
$$

are absolutely continuous, this follows now from Shale's Theorem, [Si].

By Corollary 5 we can accordingly split an arbitrary closed string to a partition of $[-\pi, \pi]$.

\section{The Bosonic Part of the Algebra (I)}

Let us illustrate the essential ideas in the simple case of the product of two fields.

Let $\widetilde{\Phi}, \widetilde{\Psi}$ be closed string bosonic fields, i.e. $L^{2}(\mu)$-functionals. For any $\widetilde{\Xi} \in L^{2}(\mu)$ we define a complex number

$$
(\tilde{\Phi}, \tilde{\Psi}, \widetilde{\Xi})_{b} \in \mathbf{C}
$$

in a continuous manner, such that we get by Riesz theorem an element $\tilde{\boldsymbol{\Phi}} * \tilde{\boldsymbol{\Psi}} \in L^{2}(\mu)$.

Let $-\pi=\sigma_{0}<\sigma_{1}=0<\sigma_{2}=\pi$ be a partition of $[-\pi, \pi]$. Due to the Corollary 5 we can split a closed string accordingly (see Fig. 1)

$$
\omega=\left(\omega^{\prime}, y\right)=\left(\omega_{1}^{\prime}, x_{1}^{\prime}, \omega_{2}^{\prime}, x_{2}^{\prime}\right)
$$

We define

$$
\begin{aligned}
(\tilde{\Phi}, \tilde{\Psi}, \tilde{\Xi})_{b^{\prime}}:= & \int d \mu_{L_{\sigma_{1}}}\left(\omega_{1}^{\prime}\right) d \mu_{L_{\sigma_{2}}}\left(\omega_{2}^{\prime}\right) d \mu_{L_{\sigma_{2}}}\left(\tilde{\omega}_{2}^{\prime}\right) d x_{1}^{\prime} d x_{2}^{\prime} d \tilde{x}_{2}^{\prime} \\
& \cdot F_{\sigma}\left(\omega_{1}^{\prime}, \omega_{2}^{\prime}, x_{1}^{\prime}, x_{2}^{\prime}\right) \tilde{\Phi}\left(\omega_{1}^{\prime}, \omega_{2}^{\prime}, x_{1}^{\prime}, x_{2}^{\prime}\right) \\
& \cdot F_{\sigma}\left(r\left(\omega_{2}^{\prime}\right), \tilde{\omega}_{2}^{\prime}, x_{2}^{\prime}, \tilde{x}_{2}^{\prime}\right) \tilde{\Psi}\left(r\left(\omega_{2}^{\prime}\right), \tilde{\omega}_{2}^{\prime}, x_{2}^{\prime}, \tilde{x}_{2}^{\prime}\right) \\
& \cdot F_{\sigma}\left(r\left(\tilde{\omega}_{2}^{\prime}\right), r\left(\omega_{1}^{\prime}\right), \tilde{x}_{2}^{\prime}, x_{1}^{\prime}\right) \tilde{\Xi}\left(r\left(\tilde{\omega}_{2}^{\prime}\right), r\left(\omega_{1}^{\prime}\right), \tilde{x}_{2}^{\prime}, x_{1}^{\prime}\right)
\end{aligned}
$$

with $r\left(\omega^{\prime}\right)(t)=\omega^{\prime}(\pi-t)$. Then we sum over all possible choices

$$
(\tilde{\Phi}, \tilde{\Psi}, \tilde{\Xi})_{b}:=\frac{1}{6}\left((\tilde{\Phi}, \tilde{\Psi}, \tilde{\Xi})_{b^{\prime}}+(\tilde{\Psi}, \tilde{\Xi}, \tilde{\Phi})_{b^{\prime}}+\cdots+(\tilde{\Xi}, \tilde{\Phi}, \tilde{\Psi})_{b^{\prime}}\right) .
$$

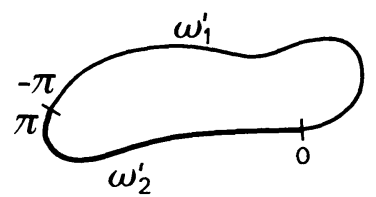

Fig. 1. 
Using Cauchy Schwartz inequality we get

$$
\left|(\tilde{\Phi}, \tilde{\Psi}, \tilde{\Xi})_{b}\right| \leqq\|\tilde{\Phi}\|_{2}\|\tilde{\Psi}\|_{2}\|\tilde{\Xi}\|_{2},
$$

where $\|\cdot\|_{2}$ denotes the $L^{2}\left(\mu_{L_{0}}\right)$-norm. By Riesz Theorem we get a unique vector denoted by

$$
[\tilde{\Phi}, \tilde{\Psi}]_{b} \in L^{2}\left(\mu_{L_{0}}\right)
$$

\section{The Restricted Polyhedra}

We want to generalize this construction to a product of $n$ closed string bosonic fields. This was suggested by M. Kaku, [K], M. Saadi and B. Zwiebach, [S-Z], and further developed by T. Kugo, H. Kunimoto, K. Suehiro, [K-K-S, K-S], A. Sen, [S], and others. The idea is to let closed string fields interact along socalled restricted polyhedra.

A restricted polyhedra is defined in $[\mathrm{K}-\mathrm{K}-\mathrm{S}, \mathrm{K}-\mathrm{S}]$ by the following properties:

i) they are homeomorphic to $S^{2}$,

ii) only three edges join at each vertex,

iii) the perimeter of any face equals $2 \pi$,

iv) any closed path (surrounding two or more faces) has a length larger or equal to $2 \pi$.

These properties are explained in the following remarks.

In string theory it was early noted that only three string fields can interact "at once." This is reflected in ii). The closed string fields are thought to give boundary conditions on the polyhedra, i.e. the perimeter of the faces are viewed as layed with closed strings. This motivates condition iii). In order to not overcount the interactions given by polyhedra one has to restrict to iv), see [S-Z, K-K-S], where the interested reader can also find the argument for including such generalized interactions.

In order to define the restricted polyhedra in an applicable way for our description of closed strings we have to take care of Lemma 1. Since we cannot use the notion of continueity, we have to rephrase i).

To define an interaction we only will use the one and zero dimensional simplices of the polyhedra.

Let $2 n$ be the number of vertices ( 0 -simplices). By condition iii) we get $3 n$ edges (1-simplices). Now the Euler number uniquely characterizes the topological sphere $S^{2}$. If our polyhedra has $(n+2)$-faces $(2$ simplices) it obeys $\mathrm{i})$.

We want to describe the moduli space of such polyhedra appropriate to the closed strings in the support of $\mu_{L_{0}}$. Let us first look at $(n+2)$-interacting closed string fields. According to the above remarks we will have $3 n$ overlapping edges and $2 n$ splitting vertices. We will describe such a configuration in the following way. In a splitting point three closed strings contact. We interpret therefore a vertex as an identification of three coordinate points of three different closed strings. In the same spirit an edge will be the identification of two parameter intervals of the interacting strings. We will put some restrictions to such choices in order to mimic the notion of restricted polyhedra.

Let us start with $2 n$ vertices. 
Let

$$
\sigma:\{1,2, \ldots, 2 n\} \rightarrow[-\pi, \pi]^{3} \times I,
$$

where $I:=\{(i, j, k) / i, j, k \in\{1,2, ., n+2\}, i \neq j \neq k \neq i\}$. The $l^{\text {th }}$ vertex is given by

$$
\sigma(l)=\left(\sigma_{1}, \sigma_{2}, \sigma_{3}, i, j, k\right)=\left(\sigma_{1}, i\right) \times\left(\sigma_{2}, j\right) \times\left(\sigma_{3}, k\right),
$$

where we say: $\sigma(l)$ splits the $i^{\text {th }}$ string in $\sigma_{1}$ etc. Condition ii) is then automatically fulfilled if we restrict to

Condition 1. Two vertices $\sigma(l), \sigma(k), l \neq k$ do not split the same string at the same point.

Next we want to describe the edges. We need some preparations.

Let

$$
S_{i}(\sigma):=\left\{\tilde{\sigma} \in[-\pi, \pi] / \exists l \in\{1,2, \ldots, 2 n\}, \sigma(l) \text { splits the } i^{\text {th }} \text { string in } \tilde{\sigma}\right\} .
$$

It is the set of splittings of the $i^{\text {th }}$ string according to $\sigma$. That the $i^{\text {th }}$ string takes part in a nontrivial interaction is reflected in

Condition 2. $\left|S_{i}(\sigma)\right| \geqq 2, i=1, ., 2, . ., n+2$.

We want to get the notion of edges. We therefore put the finite set $S_{i}(\sigma)$ in increasing order. A pair $\left(\sigma_{1}, \sigma_{2}\right) \in S_{i}(\sigma)$ is called an edge of the $i^{\text {th }}$ string iff $\sigma_{1}$ and $\sigma_{2}$ are nearest neighbors (n.n) in increasing order, or $\sigma_{1}$ is the largest and $\sigma_{2}$ the smallest element in $S_{i}(\sigma)$, (cyclic order). Thus edges are naturally directed. We denote by $E_{i}(\sigma)$ the set of edges of the $i^{\text {th }}$ string. Again we can put $E_{i}(\sigma)$ in cyclic order compatible with the order of $S_{i}(\sigma)$. To an edge $e_{i}=\left(\sigma_{1}^{i}, \sigma_{2}^{i}\right) \in E_{i}(\sigma)$ we can uniquely associate the vertices $\sigma\left(l_{1}\right), \sigma\left(l_{2}\right)$ for $\sigma_{1}, \sigma_{2}, l_{1}, l_{2} \in\{1,2, ., 2 n\}$. We denote these data by

$$
\begin{gathered}
\eta_{i}\left(e_{i}\right)=\left(l_{1}, l_{2}\right) \\
l_{1} \text { the startpoint vertex of } e_{i} \\
l_{2} \text { the endpoint vertex of } e_{i} .
\end{gathered}
$$

Also we denote by $e_{i}^{r}$ the pair $\left(\sigma_{2}^{i}, \sigma_{1}^{i}\right)$ the reversed of $e_{i}$. Now we can define an edge of the polyhedra.

A pair $\left(e_{i}, e_{j}\right) \in E_{i}(\sigma) \times E_{j}(\sigma)$ is called an edge of a polyhedra iff

i) $i \neq j$

ii) $\sigma_{2}^{i}-\sigma_{1}^{i}=\sigma_{2}^{j}-\sigma_{1}^{j}$.

The meaning of Conditions i) is obvious and ii) just says that the two parameter intervals can be identified. We remark here that the condition $\eta_{i}\left(e_{i}\right)=\eta_{j}\left(e_{j}^{r}\right)$ as would be suggested by the classical picture is not appropriate for the discontinuous strings, see Lemma 1.

Denote

$$
E(\sigma)=\bigcup_{i=1}^{n+2} E_{i}(\sigma)
$$

the set of all string edges. We want to pair two edges to one edge of the polyhedra. This is described by an involution. A map $P E$

$$
P E: E(\sigma) \rightarrow E(\sigma)
$$


is called a set of polyhedra edges, iff $(P E)^{2}=\mathrm{Id}$ and for all $e \in E(\sigma)(e, P E(e))$ is an edge of a polyhedra. Furthermore one requires that two n.n edges of a string $i$ are not mapped to n.n. edges of a string $j$.

We need a last definition. Let

$$
C=\left(e_{1}, ., e_{k}\right)
$$

be a $k$-tuple of string edges, $k \in \mathbf{N}$ and let startpoint vertex of $e_{j+1}=$ endpoint vertex of $e_{j}\left(e_{n+1}=e_{1}\right)$.

Then we call $C$ a closed path. The value

$$
\left|e_{i}\right|=\left|\left(\sigma_{1}^{i}, \sigma_{2}^{i}\right)\right|:=\sigma_{2}^{i}-\sigma_{1}^{i} \in \mathbf{R}^{+}
$$

respectively

$$
|C|:=\sum_{i=1}^{k}\left|e_{i}\right|
$$

is called the length of the closed path $C$.

Now we can transcribe all the conditions for restricted polyhedra to our case.

Condition 3. The cardinality of $E(\sigma)$ is $4 n$.

This replaces condition i) above.

Condition 4. Let $C$ be a closed path, $C=\left(e_{1}, ., e_{k}\right)$ with $e_{j+1} \neq P E\left(e_{j}\right), j=1, ., k$. Then $|C| \geqq 2 \pi$.

One easily checkes that such a closed path is a face or surrounds one or more faces of the polyhedra. This condition replaces iv).

Now we can define a restricted polyhedra to be a pair $(\sigma, P E)$ of the above type obeying conditions 1)-4). Looking at these conditions one notices that they are all linear. Hence the set $\mathscr{M}_{p}(n+2)$ of pairs $(\sigma, P E)$ as above may be described as a finite union of $(2 n-2)$-dimensional locally convex bounded subsets of $[-\pi, \pi]^{6 n}$. There is a unique measure on $\mathscr{M}_{p}(n+2)$ compatible with the convex structure, induced by the Lebesgue measure on $\mathbf{R}^{2 n-2}$. We denote this measure by $d \lambda_{\mathscr{M}}$.

\section{The Bosonic Part of the Algebra (II)}

We want to generalize the product of Chap. 3 to restricted polyhedra.

Let $(\sigma, P E)$ be a restricted polyhedra with $(n+2)$ faces and $\widetilde{\Phi}_{1}, ., \tilde{\Phi}_{n+1}, \tilde{\Psi}$ be closed string bosonic fields, i.e. $L^{2}(\mu)$-functionals. Due to the Corollary 5 we can split the $i^{\text {th }}$ closed string according to the partition $S_{i}(\sigma)$,

$$
\omega_{i}=\left(\omega_{i}^{\prime}, x_{i}^{\prime}\right)=\left(\omega_{i}^{\prime}\left(\sigma_{1}, x_{i}^{\prime}\left(\sigma_{1}\right)\right), \ldots, \omega_{i}^{\prime}\left(\sigma_{k}\right), x_{i}^{\prime}\left(\sigma_{k}\right)\right) \text {, }
$$

$k=\left|S_{i}(\sigma)\right|$. We interpret $\left(\omega_{i}^{\prime}\left(\sigma_{j}\right), x_{i}^{\prime}\left(\sigma_{j}\right)\right)$ as the range of the $j^{\text {th }}$ edge $e_{i}(j) \in E_{i}(\sigma)$. For the right-hand side we introduce the abbreviation $\omega_{i}(\sigma)$. To simplify the notation we also use the symbol $\delta$ to denote

$$
\int d \mu\left(\omega_{1}\right) d \mu\left(\omega_{2}\right) \delta\left(\omega_{1}-\omega_{2}\right) F\left(\omega_{1}, \omega_{2}\right):=\int F\left(\omega_{1}, \omega_{1}\right) d \mu\left(\omega_{1}\right)
$$

for $\mu$-measurable functions $F$, and more general we write

$$
\delta\left(e-P E(e)^{r}\right)=\delta\left(\left(\omega_{i}^{\prime}\left(\sigma_{1}\right), x_{i}^{\prime}\left(\sigma_{1}\right)\right)-\left(r\left(\omega_{j}^{\prime}\left(\sigma_{2}\right)\right), x_{j}^{\prime}\left(\sigma_{2}\right)\right)\right)
$$


if $\left(\omega_{i}^{\prime}\left(\sigma_{1}\right), x_{i}^{\prime}\left(\sigma_{1}\right)\right)$ is the range of $e \in E(\sigma)$ and $\left(\omega_{j}^{\prime}\left(\sigma_{2}\right), x_{i}^{\prime}\left(\sigma_{2}\right)\right)$ the range of $P E(e)$. Also we use the abbreviation

$$
\begin{aligned}
& F_{\sigma_{i}}\left(\omega\left(\sigma_{i}\right)\right) d \mu_{L_{\sigma_{i}}}\left(\omega\left(\sigma_{i}\right)\right) \\
& \quad=F_{\sigma_{i}}\left(\omega^{\prime}\left(\sigma_{i, 1}\right), \ldots, \omega^{\prime}\left(\sigma_{i, k}\right), x^{4}\left(\sigma_{i, 1}\right), \ldots, x^{\prime}\left(\sigma_{i, k}\right)\right) \bigotimes_{j=1}^{k} d \mu_{L_{\sigma_{t, j}}}\left(\omega^{\prime}\left(\sigma_{i, j}\right) \otimes d x^{\prime}\left(\sigma_{i, j}\right)\right),
\end{aligned}
$$

where $\sigma_{i}$ is the partition of the $i^{\text {th }}$ string according to the polyhedra $(\sigma, P E)$. For the definition of $F_{\sigma}$ see Corollary 5.

Now we are prepared to define the general product. Let $\tilde{E}(\sigma) \subset E(\sigma)$ be half of all edges such that $e \in \tilde{E}(\sigma) \Rightarrow P E(e)$ not in $\tilde{E}(\sigma)$. It corresponds to the geometric edges of the polyhedra. Then we define

$$
\begin{aligned}
\left(\tilde{\Phi}_{1}, \ldots, \tilde{\Phi}_{n+1}, \tilde{\Psi}\right)_{b^{\prime}, \sigma}:= & \int \prod_{i=1}^{n+2} F_{\sigma_{i}}\left(\omega\left(\sigma_{i}\right)\right) d \mu_{L_{\sigma_{i}}}\left(\omega\left(\sigma_{i}\right)\right) \prod_{e \in \tilde{E}(\sigma)} \delta\left(e-P E(e)^{r}\right) \\
& \cdot \tilde{\Phi}_{1}\left(\omega\left(\sigma_{1}\right)\right) \ldots \tilde{\Phi}_{n+1}\left(\omega\left(\sigma_{n+1}\right)\right) \cdot \tilde{\Psi}\left(\omega\left(\sigma_{n+2}\right)\right)
\end{aligned}
$$

and again we sum over all possible choices of putting a string on a face of the polyhedra,

$$
\left(\tilde{\Phi}_{1}, \ldots, \tilde{\Phi}_{n+1}, \tilde{\Psi}\right)_{b, \sigma}=\frac{1}{(n+2) !} \sum_{\text {permut. } i}\left(\tilde{\Phi}_{i_{1}}, \ldots, \tilde{\Phi}_{i_{n+1}}, \tilde{\Phi}_{i_{n+2}}\right)_{b^{\prime}, \sigma},
$$

where $\tilde{\Phi}_{n+2}=\tilde{\Psi}$. An iterated application of the Cauchy Schwartz inequality shows

$$
\left|\left(\tilde{\Phi}_{1}, ., \tilde{\Phi}_{n+1}, \tilde{\Psi}\right)_{b, \sigma}\right| \leqq\left(\prod_{i=1}^{n+1}\left\|\tilde{\Phi}_{i}\right\|_{2}\right)\|\tilde{\Psi}\|_{2}
$$

and by Riesz Theorem we get the definition of

$$
\left[\tilde{\Phi}_{1}, ., \tilde{\Phi}_{n+1}\right]_{b, \sigma} \in L^{2}\left(\mu_{L_{0}}\right)
$$

as the dual of the linear map in $\widetilde{\Psi}$. It is easy to see that the map

$$
(\sigma, P E) \mapsto\left[\tilde{\Phi}_{1}, ., \tilde{\Phi}_{n+1}\right]_{b, \sigma}
$$

is measurable w.r.t. $\lambda_{\mathscr{M}}$. Therefore we can define

$$
\left[\tilde{\Phi}_{1}, ., \tilde{\Phi}_{n+1}\right]_{b}:=\int_{(\sigma, P E) \in \mathscr{M}_{P}(n+2)}\left[\tilde{\Phi}_{1}, ., \tilde{\Phi}_{n+1}\right]_{b, \sigma} d \lambda_{\mathscr{M}}(\sigma) \in L^{2}\left(\mu_{L_{0}}\right) .
$$

The resulting algebra is denoted by $\mathscr{A}_{b}$.

\section{The Fermionic Part}

Before continuing the discussion some remarks on the physical interpretation of closed strings are in order. Looking at a closed string as a "closed" path in $\mathbf{R}^{d}$, i.e. as a geometrical object, one would suggest Diff $S^{1}$ as the reparametrization and thereby symmetry group of the theory. This point of view will be carried out in this paragraph.

But in the operator formalism of string theory one interprets closed strings as doubled open strings. One splits a closed string into left and right moving parts. 
The symmetry group one achieves this way is the direct product of the reparametrization groups for both parts. The slight changes we need in the construction will be explained in the next section. Let us start with the geometrical interpretation of closed strings as closed paths. In order to define things rigorously we split such a path into a based part and the zero point localization, see Sect. 2 . This restricts the reparametrizations to those leaving the zero point fixed. We get Diff $S^{1} / S^{1}$ as the symmetry group.

Let $\widetilde{\Phi}$ be a closed string bosonic field, $\gamma \in \operatorname{Diff} S^{1} / S^{1}$. The action of $\gamma$ on $\tilde{\Phi}$ is given by

$$
\gamma^{*}(\tilde{\Phi})(\omega):=\tilde{\Phi}\left(\omega \circ \gamma^{-1}\right), \quad \tilde{\Phi} \in L^{2}(\mu) .
$$

We want to compute the action of $\gamma$ on a product of such fields, at least for the moment formally.

Let $[a, b] \subset[-\pi, \pi]$ be an interval,

$$
L_{[a, b]}:=\sqrt{-\Delta_{N,[a, b]}^{\prime}}+I_{[a, b]},
$$

where $-\Delta_{N,[a, b]}$ is the Neumann Laplacian on $L^{2}([a, b])$ without zero modes, $I_{[a, b]}$ the identity on the kernel. We denote by $\mathscr{H}([a, b])$ the Sobolev space over $L^{2}([a, b])$ with weight $L_{[a, b]}^{-1}$. The eigenfunctions are explicitly given by

$$
\begin{aligned}
\psi_{[a, b], 2 n}(x) & :=\sqrt{b-a} \cos \pi n\left(\frac{2 x}{b-a}-\left(1+\frac{2 a}{b-a}\right)\right), \\
\psi_{[a, b], 2 n+1}(x) & :=\sqrt{b-a} \sin \pi n\left(\frac{2 x}{b-a}-\left(1+\frac{2 a}{b-a}\right)\right),
\end{aligned}
$$

$n \in \mathbf{Z}$, see Sect. 2 .

Given a reparametrization $\gamma \in \operatorname{Diff} S^{1} / S^{1}$ with $\gamma(a)=c, \gamma(b)=d$, we get an induced map

$$
\begin{aligned}
\gamma^{*}([c, d]): \mathscr{H}([c, d]) & \rightarrow \mathscr{H}([a, b]) \\
\omega & \mapsto L_{[a, b]}^{-1 / 2}\left(\left(L_{[c, d]}^{1 / 2} \omega \circ \gamma^{-1}\right) \cdot\left|\gamma^{\prime}\right|^{-1 / 2} \circ \gamma^{-1}\right)
\end{aligned}
$$

Let us look at the Gaussian measure $\mu_{L_{[a, b]}}$. A formal substitution leads to the expression

$$
\gamma^{*}\left(\mu_{L_{[a, b]}}\right) \approx \operatorname{det}\left(\gamma^{*}[c, d]\right) \mu_{L_{[c, d]}}
$$

It is well known that for general $\gamma$ this determinant is analytically not well defined. As in the case of open string theory, [Wie2], we will interpret the determinant as a Quillen determinant, [Se-Wi, $\mathrm{Pr}-\mathrm{Se}]$.

We want to split $L^{2}([a, b])$ into positive and negative parts. In the case of periodic boundary conditions the splitting into left and right moving parts is quite natural. We can define a square root of $-\Delta_{p}$ (periodic Laplacian) by $i \frac{d}{d x}$ with periodic boundary conditions. Then the splitting is just the splitting of the spectral spaces of $i \frac{d}{d x}$ according to positive and negative eigenvalues. In the case of Neumann boundary conditions this is not possible. But we know from Lemma 3 
that both operators are close to each other. Therefore we will approximate such a splitting.

Let

$$
\eta_{[a, b], k}:=\psi_{[a, b], 2 k}+\operatorname{sign}(k) \psi_{[a, b], 2 k+1}, \quad k \in \mathbf{Z} .
$$

These vectors form an orthonormal basis in $L^{2}([a, b])$. We define

$$
\mathscr{H}_{ \pm}([a, b]):=\overline{\operatorname{span}\left\{\eta_{[a, b], k}\right\}_{k \geqq 0, k<0}} .
$$

For another interval $[c, d] \subset[-\pi, \pi]$ we identify $\mathscr{H}([a, b])$ and $\mathscr{H}([c, d])$ by

$$
\begin{gathered}
U: \mathscr{H}([a, b]) \rightarrow \mathscr{H}([c, d]) \\
\psi_{[a, b], k} \mapsto \psi_{[c, d], k} .
\end{gathered}
$$

Obviously $U$ is unitary.

Let $\gamma \in \operatorname{Diff} S^{1} / S^{1}$ with $\gamma(a)=c, \gamma(b)=d$. Then the map

$$
U^{*} \gamma^{*}: \mathscr{H}([c, d]) \rightarrow \mathscr{H}([c, d])
$$

is unitary. Furthermore one easily proves $U^{*} \gamma^{*} \in U_{\text {res }}(\mathscr{H})$ according to the above splitting, see [Pr-Se]. We can define the Quillen determinant of that map. We therefore suggest an interpretation of (66) by replacing the analytical determinant by the Quillen determinant.

$$
\operatorname{det}\left(\gamma^{*}([a, b])\right) \rightarrow \operatorname{DET}\left(U^{*} \gamma^{*}\right) .
$$

Since the Quillen determinant is not a number in $\mathbf{C}$ but a section of the Quillen DET-bundle, see [Pr-Se], we have to work with an algebra of sections rather than an algebra of functionals, see [Wie2].

Take the holomorphic imbedding induced by (70)

$$
\imath: \operatorname{Diff} S^{1} / S^{1} \hookrightarrow \operatorname{Gr}(\mathscr{H})_{0},
$$

where $\operatorname{Gr}(\mathscr{H})_{0}$ in the identity component of the universal Grassmannian, see [Pr-Se, Mic]. We can pull back the holomorphic line bundle DET* over $\operatorname{Gr}(\mathscr{H})_{0}$, also denoted by DET*. By abuse of language let $\mathscr{A}_{b}$ also denote the trivial bundle over Diff $S^{1} / S^{1}$ with the bosonic part $\mathscr{A}_{b}$ as fibre. The new algebra consists of sections in

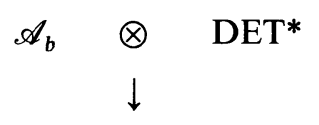

\section{Diff $S^{1} / S^{1}$.}

Next we want to lift the group action of Diff $S^{1} / S^{1}$ to an action on the bundle, thus modelling (71).

Let $\gamma \in \operatorname{Diff} S^{1} / S^{1}$ and $\rho(\gamma)$ be the natural map of the DET-fibre over $\tilde{\gamma} \in \operatorname{Diff} S^{1} / S^{1}$ into the DET-fibre over $\tilde{\gamma}^{\circ} \gamma^{-1}$. We can lift the group action of $\gamma$ to a bundle automorphism $\rho^{*}(\gamma)$ :

$$
\left(\rho^{*}(\gamma)(\Phi)\right)(\tilde{\gamma}):=\rho(\gamma)\left(\left(\gamma^{*} \cdot(\Phi)\right)\left(\tilde{\gamma}^{\circ} \gamma^{-1}\right)\right),
$$

where $\Phi$ is a section of the bundle and $\gamma^{*}$ denotes the action of $\gamma$ in the $\mathscr{A}_{b}$-fibre. 
(Notice that $\gamma \mapsto \rho(\gamma)$ is not a group homomorphism but only a projective representation, see below.)

In order to filter out the reparametrization invariant part we will mimick the cross-product construction for $C^{*}$-algebras. Since in our case there is no directly defined product, we will use Riesz theorem as above to define it.

As in the open string case, [Wie1], we will restrict to holomorphic sections of the above bundle. (Diff $S^{1 /} S^{1}$ as a flag manifold carries a natural complex structure; the imbedding $l$ into $\operatorname{Gr}(\mathscr{H})$ is holomorphic, the pull back bundle $\imath^{*}(\mathrm{DET})$ is therefore a holomorphic bundle over Diff $S^{1} / S^{1}$, see $[\mathrm{Pr}-\mathrm{Se}]$.)

On $\operatorname{Gr}(\mathscr{H})_{0}$ we have a $U_{\text {res }}(\mathscr{H})$-quasi-invariant measure $\mu_{0}$ constructed by D. Pickrell, see [Pi].

Denote

$$
\begin{aligned}
{[\mathrm{]}] \mathrm{Gr}(\mathscr{H})_{0} } & \rightarrow \operatorname{Gr}(\mathscr{H})_{0} /\left(\operatorname{Diff} S^{1} / S^{1}\right) \\
g & \mapsto[g]
\end{aligned}
$$

the canonical projection. Take a measurable section

$$
\begin{aligned}
\tau: \operatorname{Gr}(\mathscr{H})_{0} /\left(\operatorname{Diff} S^{1} / S^{1}\right) & \rightarrow \operatorname{Gr}(\mathscr{H})_{0} \\
{[g] } & \mapsto \tau([g]) .
\end{aligned}
$$

We get a Borel map

$$
\begin{aligned}
\Gamma: \operatorname{Gr}(\mathscr{H})_{0} & \rightarrow \operatorname{Diff} S^{1} / S^{1} \\
g & \mapsto \Gamma(g),
\end{aligned}
$$

where $\Gamma(g)$ is uniquely given by $g=\Gamma(g) \tau([g])$. Let $\mu^{\Gamma}$ be the induced quasiinvariant measure on Diff $S^{1} / S^{1}$. In the fiber of

$$
\begin{array}{ccc}
\mathscr{A}_{b} & \otimes & \text { DET }^{*} \\
\downarrow & \\
& \\
\text { Diff } S^{1} / S^{1} &
\end{array}
$$

we have a natural hermitian metric given by the canonical Quillen metric on DET*, [Pr-Se], and the Hilbert space structure on $\mathscr{A}_{b}$, see Sect. 1 . We define $\mathscr{H}_{\mathrm{cl}}$ to be the Hilbert space of holomorphic $L^{2}\left(\mu^{\Gamma}\right)$-sections of the bundle (73).

The product structure on $\mathscr{A}_{b}$ obviously transfers to a product structure on this fibre of the bundle. We will denote the associated multilinear forms also by $(., \ldots,,)_{b, \sigma}$ etc. Now fix a restricted $(n+2)$ polyhedra $(\sigma, P E)$. We will use the notations of Sect. 3.

For $(n+2)$ sections $\Phi_{1}, \ldots, \Phi_{n+1}, \Psi \in \mathscr{H}_{\text {cl }}$ we define

$$
\begin{array}{r}
\left(\Phi_{1}, \ldots, \Phi_{n+1}, \Psi\right)_{\sigma}:=\int_{\text {Diff } S^{1} / S^{1}} \cdots \int_{\text {Diff } S^{1} / S^{1}} d \mu^{\Gamma}\left(\gamma_{1}\right) \cdots d \mu^{\Gamma}\left(\gamma_{n+1}\right) \\
\left(\left(\rho^{*}\left(\gamma_{1}^{-1}\right) \rho^{*}\left(\gamma_{n+1}\right)\left(\Phi_{1}\right)\right)\left(\gamma_{n+1}\right), \ldots,\left(\rho^{*}\left(\gamma_{n}^{-1}\right) \rho^{*}\left(\gamma_{n+1}\right)\left(\Phi_{n}\right)\right)\left(\gamma_{n+1}\right),\right. \\
\left.\left(\rho^{*}\left(\gamma_{1}\right) \cdots \rho^{*}\left(\gamma_{n}\right)\left(\Phi_{n+1}\right)\right)\left(\gamma_{n+1}\right), \Psi\left(\gamma_{n+1}\right)\right)_{b, \sigma^{*}}
\end{array}
$$

The reparametrization invariance is then reformulated as

$$
\left(\rho^{*}(\gamma) \Phi_{1}, \ldots, \rho^{*}(\gamma) \Psi\right)_{\sigma}=\left(\Phi_{1}, ., \Psi\right)_{\sigma},
$$


$\gamma \in \operatorname{Diff} S^{1} / S^{1}$. Checking this equality we have to substitute $\gamma_{i} \rightarrow \gamma \circ \gamma_{i}$ in the integrals. Now the measure $\mu^{\Gamma}$ is not left invariant but only quasi-invariant. The densities were computed by D. Pickrell, [Pi]. They yield a projective representation of Diff $S^{1} / S^{1}$. The central charge of the associated central extension is $c=-26$.

On the other hand, as mentioned above, $\rho^{*}$ is not a representation of Diff $S^{1} / S^{1}$ as bundle automorphisms, but a projective representation. We can also compute the associated charge as $c=2 d, d$ the dimension of the target manifold of the closed strings.

Putting these results together, we can perform the substitution. As the final result we conclude the reparametrization invariance in $d=13$.

In $d=13$ we can use the invariance of the integrations. An iterated application of Cauchy-Schwarz-inequality and the finiteness of the measure $\mu^{\Gamma}$ shows

$$
\left(\Phi_{1}, \ldots, \Phi_{n+1}, \Phi\right)_{\sigma} \leqq\left\|\Phi_{1}\right\| \cdots\left\|\Phi_{n+1}\right\|\|\Psi\| .
$$

Using Riesz' theorem we get the definition of the product

$$
\left[\Phi_{1}, \ldots, \Phi_{n+1}\right]_{\sigma} \in \mathscr{H}_{\mathrm{cl}} \text {. }
$$

As in Sect. 4 we finally set

$$
\left[\Phi_{1}, \ldots, \Phi_{n+1}\right]:=\int_{\mathscr{M}_{P}(n+2)} d \lambda_{M}(\sigma)\left[\Phi_{1}, \ldots, \Phi_{n+1}\right]_{\sigma} .
$$

\section{The Closed String as a Doubled Open String}

In the operator formalism of string theory one splits a closed string into left and right moving parts.

Open strings are described by differentiable paths

$$
\omega_{\mathrm{op}}:[0, \pi] \rightarrow \mathbf{R}^{d} .
$$

As in Sect. 2 we split an open string into a based part and the localization at 0 ,

$$
\omega=\left(\omega_{\mathrm{op}}, x\right) \quad x=\omega(o) .
$$

The free dynamics of the based part is similar to the closed string case given by $(d=1$, otherwise take direct sums)

$$
L_{\mathrm{op}}=\sqrt{-\Delta_{N,[0, \pi]}^{\prime}} .
$$

The vectors

$$
\psi_{n}(x):=\psi_{[0, \pi], n}(x)=\cos n x, \quad x \in[0, \pi], n \in \mathbf{N} \backslash 0
$$

build an orthonormal basis of eigenvalues of based open strings in $L^{2}([0, \pi], d x)$. Analogously we get

$$
\sigma_{n}(x)=\sin n x, \quad \gamma_{n}(x)=\cos n x
$$

$n \in \mathbf{N} \backslash 0$ as an orthonormal basis of eigenvectors of based closed strings in $L^{2}([-\pi, \pi])$.

The splitting of a closed string into left and right moving parts is depicted in Fig. 2. 


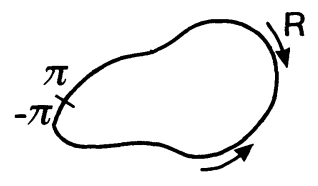

Fig. 2.

Then

$$
\begin{aligned}
& \frac{1}{\sqrt{2}}\left(\sigma_{n}+\gamma_{n}\right) \text { moves to the right } \\
& \frac{1}{\sqrt{2}}\left(\sigma_{n}-\gamma_{n}\right) \text { to the left }
\end{aligned}
$$

$n \in \mathbf{N} \backslash 0$. We therefore define the unitary map

$$
U: \mathscr{H}_{\mathrm{cl}, b} \rightarrow H_{\mathrm{op}, b} \oplus \mathscr{H}_{\mathrm{op}, b}
$$

where $\mathscr{H}_{\mathrm{cl}, b}=L^{2}([-\pi, \pi], d x) \ominus$ space $\left\{\gamma_{0}\right\}$ and $U$ is given by

$$
\begin{aligned}
& U\left(\frac{1}{\sqrt{2}}\left(\sigma_{n}+\gamma_{n}\right)\right)=\psi_{n} \oplus 0, \\
& U\left(\frac{1}{\sqrt{2}}\left(\sigma_{n}-\gamma_{n}\right)\right)=0 \oplus \psi_{n},
\end{aligned}
$$

$n \in \mathbf{N} \backslash 0$.

For a general based closed loop we get

$$
U(\omega)=\omega^{r} \oplus \omega_{l}
$$

and call $\omega^{r}, \omega^{l}$ the right and left moving part. It is fairly obvious that

$$
U^{*}\left(L_{\mathrm{ob}}^{-1} \oplus L_{\mathrm{op}}^{-1}\right) U=L_{0}^{-1}
$$

see Sect. 2. Denote

$$
d \mu_{\mathrm{op}}:=\bigoplus_{i=1}^{d} d \mu_{L_{\mathrm{op}}},
$$

where $\mu_{L_{\mathrm{op}}}$ in the Gaussian measure with covariance $L_{\mathrm{op}}$. The quantization of the based open strings in the Schrödinger picture is given by passing to the $L^{2}\left(\mu_{L_{\mathrm{op}}}\right)$ functional, see Sect. 2 . Using the above unitary equivalence of operators we get a geometrical isomorphism

$$
L^{2}(\mu) \cong L^{2}\left(\mu_{L_{0}}\right) \otimes L^{2}\left(\mathbf{R}^{d}, d x\right) \cong L^{2}\left(\mu_{L_{\mathrm{op}}}\right) \otimes L^{2}\left(\mu_{L_{\mathrm{op}}}\right) \otimes L^{2}\left(\mathbf{R}^{d}, d x\right)
$$

see [Si], induced by $U$.

This is exactly the splitting of a closed string bosonic field into left and right moving open string bosonic fields. The last factor in the above tensor product reflects the common zero point localization. Viewed as a double open string field the symmetry group of the closed string field theory could be expected as the 
direct product of two copies of the reparametrization group of the associated open strings.

Let us define the bosonic part. Using the splitting $U$ we get

$$
\mu_{L_{0}} \cong \mu_{L_{\mathrm{op}}}^{(l)} \otimes \mu_{L_{\mathrm{op}}}^{(r)}
$$

the associated splitting of the Gaussian measures.

For an arbitrary restricted polyhedra $(\sigma, P E)$ we have to replace the integration measures $\mu_{L, \sigma}$ in a proper way. We need the splitting into the left and right moving part of an arbitrary part of the closed string.

Assume $\omega$ to be closed on $[a, b]$. We split $\omega$ into left and right moving parts $\tilde{\omega}^{l}, \tilde{\omega}^{r}$ defined on $\left[\frac{b+a}{2}, b\right]$,

$$
\tilde{\omega}^{r, l}(x)=1 / 2\left(\omega\left(x+\frac{b+a}{2}\right) \pm \omega\left(x-\frac{a+b}{2}\right)\right) \quad x \in\left[\frac{a+b}{2}, b\right] \text {. }
$$
We translate the parameter interval to $\left[\frac{\pi+a}{2}, \frac{\pi+b}{2}\right]$. We use the same notation
for the translated paths. Let

$$
\begin{aligned}
L_{P}([a, b]) & :=\sqrt{-\Delta_{p,[a, b]}^{\prime},} \\
L_{\mathrm{op}}([a, b]) & :=\sqrt{-\Delta_{P,[(\pi+a) / 2,(\pi+b) / 2]}^{\prime}}
\end{aligned}
$$

Then again we get the measure decomposition

$$
\mu_{L_{P}([a, b]}=\mu_{L_{\mathrm{op}}([a, b])} \otimes \mu_{L_{\mathrm{op}}[[a, b])} .
$$

to reduce the general decomposition of a closed string to the above case one notices that by the Lemma 3

$$
\mu_{L_{P}([a, b])} \text { is absolutely continuous w.r.t. } \mu_{L_{N}([a, b])},
$$

$L_{N}([a, b]):=\sqrt{-\Delta_{N,[a, b]}^{\prime}}$. Therefore we can replace the integration measure $\mu_{L_{\sigma}}$ by tensor product measures of the form

$$
\rightarrow \mu_{L_{\mathrm{op}}([a, b])} \otimes \mu_{L_{\mathrm{op}}([a, b])}
$$

Instead of using the above isomorphism of spaces we will directly look at the. product. Using the symbolic notation of Sect. 5 we split

$$
\delta\left(e-P E(e)^{r}\right)=\delta\left(e_{l}-P E(e)_{l}^{r}\right) \cdot \delta\left(e_{r}-P E(e)_{r}^{r}\right)
$$

into left and right parts as above. But now one notices that $P E(e)_{l}^{r}$ corresponds exactly to the right moving part of $P E(e)$. We have to identify the left moving part of the first string functional with the right moving part of the second.

So we are left with the symmetry part. The reparametrization group for open string is given by Diff $[0, \pi]$. Stretching the parameter interval we can take $\operatorname{Diff}[-\pi, \pi]$. Now we again use Lemma 3 to get rid of the Neumann boundary conditions. In the string field case we replace them by periodic boundary conditions. Therefore, without loss of generality, we can take Diff $S^{1} / S^{1}$ as the symmetry group of open bosonic strings. 
On the other hand the splitting into left and right moving parts yields

$$
\mathscr{A}_{b}=\mathscr{A}_{\mathrm{op}} \otimes \mathscr{A}_{\mathrm{op}} \otimes L^{2}\left(\mathbf{R}^{d}, d x\right)
$$

with $\mathscr{A}_{\mathrm{op}}=L^{2}\left(\mu_{L_{\mathrm{op}}}\right)$, see (94). Let us only sketch how to perform the final construction. As in Sect. 6 we notice that a substitution in $\mu_{L_{\mathrm{op}}}$ according to a reparametrization is not analytically defined but categorically. As shown there we split the Hilbert space of open strings into a positive and negative part. Reparametrizing a path yields an imbedding

$$
\operatorname{Diff} S^{1} / S^{1} \hookrightarrow \operatorname{Gr}(\mathscr{H})_{0} .
$$

See (70). Pulling back the DET*-bundle and denoting by abuse of language the trivial bundle with fibre $\mathscr{A}_{\text {op }}$ also by $\mathscr{A}_{\text {op }}$ we get a holomorphic bundle

$$
\begin{aligned}
& \mathrm{DET}^{*} \otimes \mathscr{A}_{\mathrm{op}} \otimes \mathrm{DET}^{*} \otimes \mathscr{A}_{\mathrm{op}} \otimes L^{2}\left(\mathbf{R}^{d}, d x\right) \\
& \downarrow \otimes \downarrow \\
& \operatorname{Diff} S^{1} / S^{1} \quad \times \quad \operatorname{Diff} S^{1} / S^{1} .
\end{aligned}
$$

Again we have a canonical hermitian metric on that bundle. The algebra consists of $L^{2}$-holomorphic sections of that bundle w.r.t. the measure $\mu^{\Gamma} \otimes \mu^{\Gamma}$. Let $\gamma_{1} \in \operatorname{Diff} S^{1} / S^{1}$. Using the description of $\mathscr{A}_{\mathrm{op}}$ with periodic boundary conditions, see Corollary 5, we get a natural action $\gamma_{1}^{*}$ on $\mathscr{A}_{\mathrm{op}}$. Again we can lift the group action of $\gamma_{1}$ to a bundle automorphism of

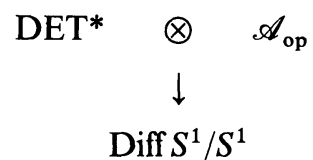

Diff $S^{1} / S^{1}$

denoted by $\rho_{1}^{*}\left(\gamma_{1}\right)$. Similarly we get a bundle lift of $\gamma_{2} \in \operatorname{Diff} S^{1} / S^{1}$ to the second bundle, denoted by $\rho_{2}^{*}\left(\gamma_{2}\right)$ and thereby a lift

$$
\rho^{*}\left(\gamma_{1} \times \gamma_{2}\right):=\rho_{1}^{*}\left(\gamma_{1}\right) \times \rho_{2}^{*}\left(\gamma_{2}\right)
$$

to the total bundle. One easily transcripts the definition of products in Sect. 6 to this doubled case. Again, by the same methods one proves the symmetry invariance for $d=26$. It is a matter of routine to fill in the details.

The symmetry $S^{1}$ of translating the common zero point localization causes no trouble. It is a compact group and integration w.r.t. the Haar measure yields the final results above.

\section{The sh-Lie-Algebra-Structure}

Let us reformulate the theory of closed string fields in more familiar terms. Instead of working with Gaussian processes on path space we isomorphically could work with processes on Fock space, [Si].

Let us look at the bosonic part $L^{2}\left(\mu_{L_{0}}\right)$. As it is well known this part is isomorphic in a natural way to the closed string Fock space generated by the 
creation and annihilation operators $\left(a_{n}^{i}\right)^{*}$ respectively $\left(a_{n}^{o}\right) i=1, \ldots, 13, n \in \mathbf{Z} \backslash 0$,

$$
\begin{aligned}
{\left[\left(a_{n}^{i}\right)^{*},\left(a_{m}^{j}\right)\right] } & =\delta_{i, j} \delta_{n, m}|n| \\
{\left[\left(a_{n}^{i}\right),\left(a_{m}^{j}\right)\right] } & =\left[\left(a_{n}^{i}\right)^{*},\left(a_{m}^{j}\right)^{*}\right]=0 .
\end{aligned}
$$

To incorporate the $L^{2}\left(\mathbb{R}^{13}, d x\right)$-factor we decompose

$$
L^{2}\left(\mu_{0}\right)=L^{2}\left(\mu_{L_{0}}\right) \otimes L^{2}\left(\mathbb{R}^{13}\right)=\int_{\mathbb{R}^{13}} L^{2}\left(\mu_{L_{0}}\right)(p) d p
$$

where $p$ is the momentum variable, conjugate to the midpoint localization, and $L^{2}\left(\mu_{L_{0}}\right)(p)$ is the Fockspace with the additional operator $\left(a_{0}^{i}\right)^{*}=a_{0}^{i} i=1, ., 13$,

$$
\left(a_{0}^{i}\right) * \Omega_{\mathrm{cl}}=p^{i} \Omega_{\mathrm{cl}}
$$

where $p \in \mathbb{R}^{13}, \Omega_{\mathrm{cl}}$ the vacuum of the Fockspace. We denote this representation of $L^{2}\left(\mu_{L_{0}}\right)$ by $\mathscr{F}_{\mathrm{cl}}$.

For the fermionic part we need the following slight extension.

We pulled back the DET*-bundle from $\operatorname{Gr}(\mathscr{H})_{0}$ to Diff $S^{1} / S^{1}$. But we should better work on $\operatorname{Gr}(\mathscr{H})_{0}$ directly. A proper interpretation for this is given as follows. There is a whole family of complex structures on Diff $S^{1} / S^{1}$ parametrized by the way one splits the tangent space into positive respectively negative, i.e. holomorphic and antiholomorphic parts, see [Wie 1]. In this way one should think of $\operatorname{Gr}(\mathscr{H})_{0}$ as a space of such splittings and $\operatorname{Gr}(\mathscr{H})_{0} / \operatorname{Diff} S^{1} / S^{1}$ is a parameter space of complex structures on Diff $S^{1} / S^{1} . \operatorname{Gr}(\mathscr{H})_{0}$ is a proper analog of a twistor space, [Pen]. The Borel isomorphism

$$
\begin{aligned}
\operatorname{Gr}(\mathscr{H})_{0} & \rightarrow\left(\operatorname{Gr}(\mathscr{H}) /\left(\operatorname{Diff} S^{1} / S^{1}\right)\right) \times \operatorname{Diff} S^{1} / S^{1} \\
g & \mapsto([g], \Gamma(g))
\end{aligned}
$$

yields a natural groupoid structure on $\operatorname{Gr}(\mathscr{H})$ :

$$
g_{1} \perp g_{2}:=\left(\left[g_{1}\right], \Gamma\left(g_{1}\right) \circ \Gamma\left(g_{2}\right)\right),
$$

$g_{1}, g_{2} \in \operatorname{Gr}(\mathscr{H})$ with $\left[g_{1}\right]=\left[g_{2}\right]$, and a natural groupoid action on $\mathscr{A}_{b}$ :

$$
g(\tilde{\Phi}):=\Gamma(g)^{*}(\tilde{\Phi})
$$

$\tilde{\Phi} \in \mathscr{A}_{b}$.

The generating elements of the closed string field algebra are tentatively given as the holomorphic $L^{2}\left(\mu_{0}\right)$-sections of

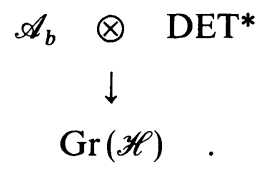

In defining the products we need some slight changes. The symmetry group Diff $S^{1} / S^{1}$ is replaced by the groupoid $\operatorname{Gr}(\mathscr{H})$. This is achieved by the following substitutions

$$
\begin{aligned}
\operatorname{Diff} S^{1} / S^{1} & \rightarrow \operatorname{Gr}(\mathscr{H})_{0}, \\
\Phi(\gamma) & \rightarrow \Phi([g], \Gamma(g)), \\
\rho^{*}(\gamma) & \rightarrow \rho^{*}([g], \Gamma(g)):=\rho^{*}(\Gamma(g)),
\end{aligned}
$$




$$
\begin{aligned}
d \mu^{\Gamma}\left(\gamma_{1}\right) \cdots d \mu^{\Gamma}\left(\gamma_{n+1}\right) \rightarrow & d \mu_{0}\left(( [ g _ { 1 } ] , \Gamma ( g _ { 1 } ) ) \cdots d \mu _ { 0 } \left(\left(\left[g_{n+1}\right], \Gamma\left(g_{n+1}\right)\right)\right.\right. \\
& \cdot \prod_{j=2}^{n+1} \delta\left(\left[g_{1}\right]-\left[g_{j}\right]\right) .
\end{aligned}
$$

It is not difficult to carry out the details.

The closed string fields are as remarked tentatively given by the holomorphic $L^{2}\left(\mu_{0}\right)$-sections of the above bundle. The bundle $\mathscr{A}_{b}$ is trivial and we can rewrite this Hilbert space as a tensor product of $\mathscr{A}_{b}$ with the Hilbert space $\Lambda_{\infty}^{*}$ of holomorphic $L^{2}\left(\mu_{0}\right)$-sections of the DET*-bundle. Using the isomorphism of D. Pickrell, [Pi], this later Hilbert space can be identified with the fermionic string Fock space. Let $\left\{L_{n}\right\}$ be the usual basis of the Witt algebra

$$
\left[L_{n}, L_{m}\right]=(m-n) L_{m+n} \quad m, n \in \mathbf{Z}
$$

which is the well known complexified Lie-algebra of Diff $S^{1} / S^{1}$. Defining the $L_{n}$ as an orthonormal basis we get a Hilbert space decomposition

$$
\mathscr{H}_{+}=\overline{\operatorname{span}\left\{L_{n}\right\}_{n} \geqq 0}=\mathscr{H}_{+} \oplus \mathscr{H}_{-} . \overline{\operatorname{span}\left\{L_{n}\right\}_{n<0}} .
$$

The splitting corresponds obviously to the various splittings into positive and negative spectral parts used above. The fermionic Hilbert space in string theory is defined as

$$
\Lambda^{*} \mathscr{H}_{+} \wedge\left(\Lambda^{*} \mathscr{H}_{-}\right)^{*}=: \mathscr{F}_{\text {gh }},
$$

see $[\mathrm{Pr}-\mathrm{Se}, \mathrm{F}-\mathrm{G}-\mathrm{Z}]$. We define the fermionic creation and annihilation operator,

$$
\begin{array}{cl}
b^{*}(n):=L_{n} \wedge & n \geqq 0\left(\text { exterior multiplication with } L_{n}\right) \\
b(-n):=L_{-n}^{*} \wedge & \left.n<0 \text { (exterior multiplication with } L_{-n}\right)
\end{array}
$$

and by the adjoint on $\Lambda^{*} \mathscr{H}_{+} \wedge\left(\Lambda^{*} \mathscr{H}_{-}\right)^{*}$

$$
\begin{aligned}
b^{*}(-n) & :=(b(-n))^{*} & & n>0, \\
b(n) & :=(b(n))^{*} & & n \leqq 0 .
\end{aligned}
$$

These are bounded operators on $\Lambda^{*} \mathscr{H}_{+} \wedge\left(\Lambda^{*} \mathscr{H}_{-}\right)^{*}$. We define

$$
\operatorname{deg}\left(b^{*}\right)=1, \quad \operatorname{deg}(b)=-1 .
$$

In this way we get a Z-graded Hilbert space. This grading is called the ghost number. D. Pickrell defined an explicit isomorphism, [Pi, Wie1]

$$
\Lambda_{\infty}^{*} \cong\left(\Lambda^{*} \mathscr{H}_{+} \wedge\left(\Lambda^{*} \mathscr{H}_{-}\right)^{*}\right)_{0}
$$

where the 0 denotes the zero graded subspace. Therefore our space of closed fields consists only of the physical closed string fields with zero ghost number. How to enlarge the space in order to get all physical fields is not difficult, [Wie1]. We sketch it in the Appendix.

Let us present the essentials. The final Hilbert space will be naturally isomorphic to

$$
\mathscr{F}:=\mathscr{F}_{\mathrm{cl}} \otimes \mathscr{F}_{g h}
$$


Denote $\Omega$ the vacuum. The subspace $\mathscr{F}_{0}$ of finitely many excitations is then given by the algebraic space of vectors

$$
\psi=\prod_{i=1}^{k} a_{-n_{i}}^{v_{i}} \otimes \bigwedge_{i=1}^{l} L_{s_{i}} \wedge \bigwedge_{i=1}^{m} L_{-r_{i}}^{*} \wedge \Omega
$$

with $k, l, m \in \mathbf{N}, v_{i} \in\{1, . ., 26\}, n_{i}, r_{i} \in \mathbf{N} \backslash\{0\}, s_{i} \in \mathbf{N}$.

It is a dense subspace of $\mathscr{F}_{\mathrm{cl}} \otimes \mathscr{F}_{g h}$. The symmetry action of Diff $S^{1} / S^{1}$ on the Hilbert space $\mathscr{A}_{b}$ leads to an infinitesimal action on $\mathscr{F}_{0}$ given by

$$
\pi\left(L_{n}\right):=\sum_{m \in \mathbf{Z}} \sum_{i=1}^{13}\left(a_{m}^{i}\right) * a_{n-m}^{i} \otimes \operatorname{Id}_{\mathscr{F}_{\mathrm{c} 1}}
$$

the symmetry action on $\left(\Lambda^{*} \mathscr{H}_{+} \wedge\left(\Lambda^{*} \mathscr{H}_{-}\right)^{*}\right)_{0}$ to an action

$$
\rho\left(L_{n}\right):=\operatorname{Id}_{\mathscr{F}_{\mathrm{c} 1}} \otimes\left(\sum_{m \in \mathbf{Z}}(m-n): b^{*}(m) b(m+n):\right)
$$

with : : the usual wick ordering.

From [Wie1] we infer

Theorem 6. Let

$$
d:=\sum_{n \in \mathbf{Z}} \pi\left(L_{n}\right) b^{*}(n)+\sum_{m<n}(m-n): b(m+n) b^{*}(m) b^{*}(n):
$$

on $\mathscr{F}_{0}$. Then $d$ is closable. We define

$$
Q:=\text { closure of } d \text {. }
$$

For the proof see [Wie1]. $Q$ is nothing but the BRST-operator in physics. Mathematically one would think of $Q$ as a Lie-coboundary of the Witt-algebra with values in the module $\mathscr{A}_{b}$, see below.

Now we can formulate the main

Theorem 7. Let $\Phi_{1}, \ldots, \Phi_{N+1} \in \mathscr{F}_{0}$. Then $\left[\Phi_{1}, \ldots, \Phi_{N+1}\right] \in D(Q)$. For $\Phi_{j}$ with fixed ghost numbers we get

$$
Q\left[\Phi_{1}, \ldots, \Phi_{N+1}\right]-\sum_{j=1}^{N+1}(-1)^{n(j)}\left[\Phi_{1}, \ldots, Q \Phi_{j}, . ., \Phi_{N+1}\right]=0
$$

where $n(j):=\sum_{i=1}^{j-1} \operatorname{deg}\left(\Phi_{i}\right)$.

Before proving this theorem we need some preparations.

We use the picture of closed string fields given as sections of the bundle (73). The elements in $\mathscr{F}_{0}$ correspond to holomorphic sections of the bundle (73) with values in the polynomial algebra over path space. Using either the Fock space picture or the section picture it is obvious that these vectors are in the domain of $Q^{n}$ for all $n \in \mathbf{N}$. In particular the set of infinitely differentiable (w.r.t. the groupoid action) sections is dense in $\mathscr{F}_{\mathrm{cl}} \otimes \mathscr{F}_{\text {gh }}$. To proceed further, we need a more detailed description of $Q$ acting on sections.

Fix a parametrization $\theta$ of $S^{1}$. We have a global section $\frac{d}{d \theta}$ in the tangent bundle. Let $v=v(\theta) \frac{d}{d \theta}$ be a $C^{\infty}$-vector field on $S^{1}$. It can be viewed as a Lie-algebra 
element of Diff $S^{1} / S^{1}$, [Mil]. The exponentiation yields the one parameter group $\gamma_{v} \in \operatorname{Diff} S^{1} / S^{1}$

uniquely defined through

$$
\frac{d}{d \theta} \gamma_{v}(\theta)_{\mid \theta=0}=v, \quad \gamma_{v}(0)=1 .
$$

Restricting on vector fields with $v(1)=0$ we get a one parameter group in Diff $S^{1} / S^{1}$. The later group is identified with the subgroup of Diff $S^{1} / S^{1}$ fixing the $1 \in S^{1}$.

Using this geometrical point of view we can describe $Q$ as follows. Given such a vector field $v$ and a section $\Phi_{\in} \mathscr{F}_{0}$,

$$
\rho^{*}\left(\gamma_{v}(\theta)\right)(\Phi)
$$

is well defined, see Sect. 6. In this picture $Q$ is a map

$$
Q: T_{1} \operatorname{Diff} S^{1} / S^{1} \rightarrow \mathscr{B}\left(\mathscr{F}_{0}, \mathscr{F}_{0}\right),
$$

where $\mathscr{B}\left(\mathscr{F}_{0}, \mathscr{F}_{0}\right)$ denotes the space of linear maps. Explicitly

$$
Q(v)(\Phi):=\frac{d}{d \theta} \rho^{*}\left(\gamma_{v}(\theta)\right)(\Phi)_{\mid \theta=0} .
$$

In the Fock space picture this can be identified with

$$
Q(v)(\Phi)=\sum_{n \in \mathbb{N}} v_{n} b(n) Q \Phi+\sum_{n \in \mathbb{N} \backslash 0} v_{-n} b^{*}(-n) Q \Phi
$$

with

$$
\begin{aligned}
v & =\sum_{n \in \mathbb{N}} v_{n} L_{n}, \quad L_{n}=e^{i n \theta} \frac{d}{d \theta} \\
v_{n} & =\int_{s^{1}} v(\theta) e^{i n \theta} d \theta .
\end{aligned}
$$

From the geometrical point of view it is now easy to see

Lemma 8. Let $\Phi_{1}, \ldots, \Phi_{N+1} \in \mathscr{F}_{0}$. Then for a $C^{\infty}$-vector field $v$ as above

$$
Q(v)\left(\left[\Phi_{1}, \ldots, \Phi_{N+1}\right]\right)=\frac{d}{d \theta} \rho^{*}\left(\gamma_{v}(\theta)\right)\left(\left[\phi_{1}, . ., \phi_{N+1}\right]\right)_{\mid \theta=0} \in \mathscr{F}_{\mathrm{cl}} \otimes \mathscr{F}_{g h} .
$$

is well defined.

Proof. A simple application of Lebesgue-Theorem of majorized convergence.

For $\Phi \in \mathscr{F}_{0}$ we easily get by explicit calculation

$$
\|Q(v)(\Phi)\|^{2} \leqq \sum_{n \in \mathbf{Z}}\left|v_{n}\right|^{2} \cdot \text { const. }
$$

In order to prove $\left[\Phi_{1}, \ldots, \Phi_{N+1}\right] \in D(Q)$ one would like to have an estimation

$$
\left\|Q(v)\left(\left[\Phi_{1}, \ldots, \Phi_{N+1}\right]\right)\right\|^{2} \leqq \sum_{n \in \mathbf{Z}}\left|v_{n}\right|^{2} \cdot \text { const. }
$$


Then one would get the well defined expression

$$
\begin{aligned}
Q\left(\left[\Phi_{1}, \ldots, \Phi_{N+1}\right]\right)= & \sum_{n \in \mathbf{N}} b^{*}(n) Q\left(L_{n}\right)\left(\left[\Phi_{1}, \ldots, \Phi_{N+1}\right]\right) \\
& +\sum_{n \in \mathbf{N} \backslash 0} b(-n) \Phi\left(L_{n}\right)\left(\left[\Phi_{1}, \ldots, \Phi_{N+1}\right]\right) \in \mathscr{F}_{\mathrm{cl}} \otimes \mathscr{F}_{g h} .
\end{aligned}
$$

We will not prove such an estimate directly. Instead we will work with $Q(v)$ and prove an equation analogous to the one in Theorem 7 . From this equation one easily gets such an estimate and also the final proof of the theorem.

Theorem 9. Let $v$ be a $C^{\infty}$-vector field on $S^{1}$ as above, $\Phi_{1}, \ldots, \Phi_{N+1}$ closed string fields in $\mathscr{F}_{0}$ with fixed ghost numbers. Then one gets

$$
Q(v)\left(\left[\Phi_{1}, \ldots, \Phi_{N+1}\right]\right)-\sum_{i=1}^{N+1}(-1)^{n(j)}\left[\Phi_{1}, . ., Q(v) \Phi_{j}, . ., \Phi_{N+1}\right]=0 .
$$

Corollary 10. For $\Phi_{1}, \ldots, \Phi_{N+1} \in \mathscr{F}_{0}$ we get $\left[\Phi_{1}, \ldots, \Phi_{N+1}\right] \in D(Q)$.

Proof. From the above equation and estimation (137) one gets easily an estimation of the form (138). The above remarks show Corollary 10.

Proof of Theorem 9. Let $\Psi \in \mathscr{F}_{0}$. We will prove

$$
\left\langle\left[\Phi_{1}, \ldots, \Phi_{N+1}, Q(v) \Psi\right]\right\rangle=\sum_{j=1}^{N+1}(-1)^{n(j)}\left\langle\left[\Phi_{1}, \ldots, Q(v) \Phi_{j}, \ldots, \Phi_{N+1}\right], \Psi\right\rangle .
$$

From this equation Theorem 9 follows.

To prove Eq. $(140)$ let $(\sigma, P E)$ be a restricted polyhedra. From the discussion in Sect. 5 we know that

$$
\left(\Phi_{1}, \ldots, \Phi_{N+1}, \rho^{*}(\gamma) \Psi\right)_{\sigma}=\left(\rho^{*}\left(\gamma^{-1}\right) \Phi_{1}, \ldots, \rho^{*}\left(\gamma^{-1}\right) \Phi_{N+1}, \Psi\right)_{\sigma},
$$

$\gamma \in \operatorname{Diff} S^{1} / S^{1}$. We get

$$
\begin{aligned}
& \left(\Phi_{1}, \ldots, \Phi_{N+1}, \rho^{*}\left(\gamma_{v}(\theta)\right) \Psi\right) \\
& \quad=\int_{\mathscr{M}_{p}(N+2)} d \lambda_{\mathscr{M}}(\sigma)\left(\rho^{*}\left(\gamma_{v}(-\theta)\right) \Phi_{1}, \ldots, \rho^{*}\left(\gamma_{v}(-\theta)\right) \Phi_{N+1}, \Psi\right)_{\sigma}
\end{aligned}
$$

and taking derivatives

$$
\left(\Phi_{1}, \ldots, \Phi_{N+1}, Q(v) \Psi\right)=\sum_{i=1}^{N+1}(-1)^{n(j)}\left(\Phi_{1}, \ldots, Q(v) \Phi_{j}, \ldots, \Phi_{N+1}, \Psi\right)
$$

from which (140) follows.

Let us pass to the physical interpretation of a closed string as a doubled open string.

As remarked in Sect. 6 the symmetry group in this case is Diff $S^{1} / S^{1} \times$ Diff $S^{1} / S^{1} \times S^{1}$. The Diff $S^{1} / S^{1}$-parts are managed as above. But due to the third factor we get additional terms.

Let us introduce the Fockspace notations. The open string bosonic Fockspace is generated by the operators $\left(a_{n}^{i}\right)^{*}, a_{n}^{i}, i=1, \ldots, 26, n \in \mathrm{N}$ with the same commutation relations as in the closed string case. To distinguish between the left and right moving parts we mark the creation and annihilation operators of the second by 
$\overline{\left(a_{n}^{i}\right)^{*},\left(a_{n}^{i}\right)}$. The constraint of equal zero point localization means $a_{0}^{i}=\bar{a}_{0}^{i}, i=1, \ldots, 26$. The fermionic or symmetry Fock space is also doubled and we mark the different parts also by $a^{-}$. But we have an additional $S^{1}$-symmetry generated by $L_{0}-\bar{L}_{0}$. Therefore in this case the BRST-operator is slightly changed.

Let

$$
\mathscr{H}_{\text {phys }}:=\mathscr{F}_{\text {op }} \otimes \overline{\mathscr{F}}_{\text {op }} \otimes \mathscr{F}_{\text {gh }} \otimes \overline{\mathscr{F}}_{\text {gh }}
$$

and $\mathscr{H}_{\text {phys }, 0}$ the subspace of finite excitations, see above. On $\mathscr{H}_{\text {phys }, 0}$ we define

$$
d_{\mathrm{phys}}:=d+\bar{d}+\frac{1}{2}\left(\pi\left(L_{0}\right)+\rho\left(L_{0}\right)-\pi\left(\bar{L}_{0}\right)-\rho\left(\bar{L}_{0}\right)\right) .
$$

The last summand is the generator of the $S^{1}$-symmetry. As in [Wie1] we get

Lemma 11. The operator $d_{\mathrm{phys}}$ is closable. Denote $Q_{\mathrm{phys}}$ the closure.

Now we can prove the sh-Lie-algebra-structure of the algebra.

Theorem 12. Let $\Phi_{1}, \ldots, \Phi_{N+1} \in \mathscr{H}_{\text {phys }, 0}$. Then $\left[\Phi_{1}, \ldots, \Phi_{N+1} \in D\left(Q_{\mathrm{phys}}\right)\right.$. Assume now that the $\Phi_{i}$ have fixed ghost numbers $d_{e}(i), d_{r}(i)$ for left and right moving parts. Then

$$
\begin{aligned}
& Q_{\mathrm{phys}}\left[\Phi_{1}, \ldots, \Phi_{N+1}\right]-\sum_{j=1}^{N+1}(-1)^{n(j)}\left[\Phi_{1}, \ldots, Q_{\mathrm{phys}} \Phi_{j}, \ldots, \Phi_{N+1}\right] \\
& =\sum_{m+n=N, m \leqq n \text { partitions } i, j}(-1)^{\sigma(i, j)} \frac{1}{2}(b(o)-b(\bar{o}))\left(\left[\left[\Phi_{i_{1}}, \ldots, \Phi_{i_{m}}\right], \Phi_{j_{1}}, \ldots, \Phi_{j_{m}}\right]\right),
\end{aligned}
$$

where $\sigma(i, j)=s_{l}(i) s_{r}(j), n(j)=\sum_{k=1}^{j_{1}} d_{e}(k)+d_{r}(k)$ and $s_{l}, s_{r}$ are computed as the s-value (158) in the appendix w.r.t. the ghost numbers of the left and right parts.

Proof. From Theorem 7 one gets for $\tilde{Q}:=Q_{\text {phys }}-\frac{1}{2}\left(\pi\left(L_{0}\right)-\rho\left(L_{0}\right)-\pi\left(\bar{L}_{0}\right)+\rho\left(\bar{L}_{0}\right)\right)$,

$$
\begin{gathered}
{\left[\Phi_{1}, \ldots, \Phi_{N+1}\right] \in D(\tilde{Q}),} \\
\tilde{Q}\left(\left[\Phi_{1}, \ldots, \Phi_{N+1}\right]\right)=\sum_{j=1}^{N+1}(-1)^{n(j)}\left[\Phi_{1}, \ldots, \tilde{Q} \Phi_{j}, \ldots, \Phi_{N+1}\right] .
\end{gathered}
$$

So the difference in the formula (147) w.r.t. (140) results from the $S^{1}$ symmetry. We are left with the computation of

$$
\left(\pi\left(L_{0}\right)+\rho\left(L_{0}\right)-\pi\left(\bar{L}_{0}\right)-\rho\left(\bar{L}_{0}\right)\right)\left(\left[\Phi_{1}, \ldots, \Phi_{M+1}\right]\right)
$$

For this we have to look at the action of $S^{1}$ on the moduli space $\mathscr{M}_{P}(N+2)$ of restricted polyhedra. But his action is induced by the translations in $S^{1}$. The infinitesimal action of $S^{1}$ leads therefore to an exterior derivation w.r.t. to the $\sigma$-variable. Now we can use Stoke's theorem. We know from the very definition of the moduli space $\mathscr{M}_{P}(N+2)$ that the boundary $\partial \mathscr{M}$ is piecewise affine. We get for the additional part

$$
\int_{\partial \mathscr{M}_{P}(N+2)} d \lambda_{\partial \mathscr{M}}\left(\sigma^{\prime}\right)(b(o)+b(\bar{o}))\left(\left[\Phi_{1}, \ldots, \Phi_{N+2}\right]_{\sigma^{\prime}}\right) .
$$

The boundary of the moduli space consists of glueing patterns, where at least one edge of a restricted polyhedra passes to 0 or $\pi$; see Fig. 3 . 


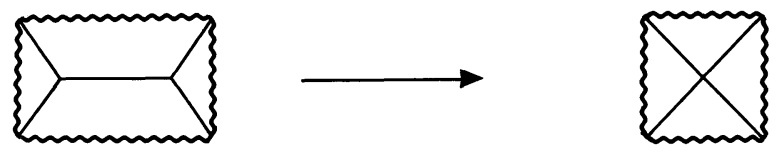

Fig. 3.

By Stoke's theorem the additional term results from the patterns with only one edge passing to an extremal. Such a pattern can be described as follows. One splits the configuration into the restricted polyhedra which are glued along one face: see Fig. 4.
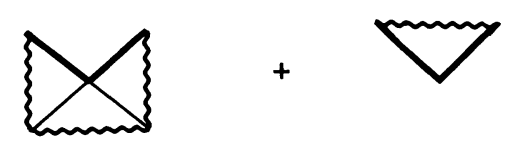

Fig. 4.

We get for the additional term

$$
\sum_{m+n=N, m \leqq n} \sum_{\text {partition } i, j}(-1)^{\sigma(i, j)} \frac{1}{2}(b(o)-b(\bar{o}))\left(\left[\left[\Phi_{i_{1}}, \ldots, \Phi_{j_{m}}\right], \Phi_{j_{1}}, \ldots, \Phi_{j_{m}}\right]\right),
$$

where $\sigma(i, j)$ results from different orderings in the product. Therefore we get the final proof.

\section{Appendix}

In this appendix we will show how to enlarge the string algebra in order to incorporate fields with nonzero ghost numbers.

Let $L_{n}, \mathscr{H}$ etc. be as in Sect. 6. We define the shift operator

$$
S: \mathscr{H} \rightarrow \mathscr{H}
$$

given by $S\left(L_{n}\right):=L_{n+1}$. Let $\operatorname{Gr}(\mathscr{H})_{n}$ be the connectivity component of $\operatorname{Gr}(\mathscr{H})$ with index $n, n \in \mathbf{N}$. Then one maps holomorphically

$$
S^{n}:=\operatorname{Gr}(\mathscr{H})_{0} \rightarrow \operatorname{Gr}(\mathscr{H})_{n} .
$$

We can pull back the DET*-bundle over $\operatorname{Gr}(\mathscr{H})_{n}$ to a bundle $\operatorname{DET}_{n}^{*}$ over $\operatorname{Gr}(\mathscr{H})$. As the final elements of our algebra we take holomorphic $L^{2}$-sections in

$$
\begin{gathered}
\mathscr{A}_{b} \otimes \bigoplus_{n \in \mathbf{Z}} \mathrm{DET}_{n}^{*} \\
\downarrow \\
\operatorname{Gr}(\mathscr{H}) .
\end{gathered}
$$

This Hilbertspace is isomorphic via the Pickrell map to $\mathscr{F}_{g h}$. We defined the product 
for zero charged sections. Using the shift operator $S$ we can identify the bundles $\mathrm{DET}_{n}^{*} \cong \mathrm{DET}_{0}^{*}$ over $\operatorname{Gr}(\mathscr{H})$. Especially for sections $\Phi_{i}$ with charge $\operatorname{deg}\left(\Phi_{i}\right)=d(i)$ we can define the product

$$
\left(\Phi_{1}, \ldots, \Phi_{N+2}\right)_{b^{\prime}, \sigma}
$$

where $(\cdot)_{b^{\prime}, \sigma}$ denotes the product without summing over all possibilities for putting the strings on some face of the polyhedra, see Sect. 5 .

Now we take care of the degree. We define

$$
\left(\Phi_{1}, \ldots, \Phi_{N+2}\right)_{b, \sigma}:=\frac{1}{(N+2) !} S^{-\sum_{i} d(i)} \sum_{\text {permutations } j}(-1)^{\operatorname{sign}(j)}\left(\Phi_{j_{1}}, \ldots, \Phi_{j_{N+2}}\right)_{b^{\prime}, \sigma}
$$

with

$$
s(j)= \pm 1
$$

computed in the following way. For $j$ the transposition $(i, i+1)$ one puts $s(j)=1$ for $d(i) \bmod 2$ and $d(i+1) \bmod 2$ both $1, s(j)=0$ otherwise. On products of permutations $s$ is defined as the product of the values on the factors. It is not hard to prove that $s$ is well defined. This definition replaces the special one in Sect. 5.

Acknowledgement. This work was initiated by the paper [St2] of J. Stasheff whom I want to thank for sending me the manuscript prior to publication. Also he pointed out to me that the appropriate name of the algebra structure should be a sh-Lie-algebra structure. I would like to thank F. Nill for many stimulating discussions.

\section{References}

[S-Z] Saadi, M., Zwiebach, B.: Closed string field theory from polyhedra. Ann. Phys. 192, 213 (1989)

[K] Kaku, M.: Geometric derivation of string field theory from first principles: Closed strings and modular invariance. Phys. Rev. D38, 3052 (1988)

[K-K-S] Kugo, T., Kunimoto, H., Suehira, K.: Non-polynomial closed string field theory. Phys. Lett. 226B, 48 (1989)

[S] Sen, A.: On the background independence of string field theory. Preprint TIFR/TH/90-7

[St1] Stasheff, J.: An almost groupoid structure for the space of (open) strings and implications for string field theory. In: Advances in Homotopy Theory, LMS Lecture Note Series 139, 165 (1989)

[St2] Stasheff, J.: Towards a closed string field theory: Topology and convolution algebra. Preprint UNC-MATH-90/1

[Co-La] Collela, P., Lanford, O.: Sample field behavior for the free markov random field. In: Constructive quantum field theory. Velo, G., Wightman, A. (eds.). Lecture Notes in Physics Vol. 25, Berlin, Heidelberg, New York: Springer 1973

[R-S IV] Reed, M., Simon, B.: Methods of modern mathematical physics. Vol. IV, New York: Academic Press 1979

[G-R-S] Guerra, F., Rosen, L., Simon, B.: Boundary conditions in $P(\Phi)_{2}$ Euclidean field theory. Ann. l'Inst. H. Poincaré 115, 231 (1976)

[Si] Simon, B.: Functional integration and quantum physics. New York: Academic Press 1979

[Se] Segal, G.: Unitary representation of some infinite dimensional groups. Commun. Math. Phys. 80, 301 (1981)

[Pr-Se] Pressley, A., Segal, G.: Loop groups. Oxford: Clarendon Press 1986

[Mic] Mickelsson, J.: String Quantization on Group Manifolds and the Holomorphic Geometry of Diff $S^{1} / S^{1}$. Commun. Math. Phys. 112, 653 (1987) 
[Wie1] Wiesbrock, H.-W.: The $C^{*}$-algebra of Bosonic Strings. Commun. Math. Phys. 136, 369-397 (1991)

[Wie2] Wiesbrock, H.-W.: A Note on the construction of the $C^{*}$-algebra of Bosonic strings. To be published in J. Math. Phys.

[Pi] Pickrell, D.: Measures on infinite dimensional Grassmann Manifolds. J. Funct. Anal. 70, 357 (1987)

[Pen] Penrose, R.: The twistor programme. Rep. Math. Phys. 12, 65 (1977)

[F-G-Z] Frenkel, I., Garland, H., Zuckerman, G.: Semi-infinite dimensional cohomology and string theory. Proc. Nat. Acad. Sci. USA 83, 844 (1986)

[Mil] Milnor, J.: Remarks on infinite dimensional Lie-groups. In: Relativity, groups and topology II. Les Houches Session XL, de Witt, B., Stora, R. (eds.). (1983)

Communicated by N. Yu. Reshetikhin 